\title{
Ultrastructural and transcriptional profiling of neuropathological misregulation of CREB function
}

\author{
LM Valor ${ }^{*, 1}$, D Jancic ${ }^{1}$, R Lujan ${ }^{2}$ and A Barco ${ }^{*, 1}$
}

We compare here the neurodegenerative processes observed in the hippocampus of bitransgenic mice with chronically altered levels of cAMP-response element-binding protein (CREB) function. The combination of genome-wide transcriptional profiling of degenerating hippocampal tissue with microscopy analyses reveals that the sustained inhibition of CREB function in A-CREB mice is associated with dark neuron degeneration, whereas its strong chronic activation in VP16-CREB mice primarily causes excitotoxic cell death and inflammation. Furthermore, the meta-analysis with gene expression profiles available in public databases identifies relevant common markers to other neurodegenerative processes and highlights the importance of the immune response in neurodegeneration. Overall, these analyses define the ultrastructural and transcriptional signatures associated with these two forms of hippocampal neurodegeneration, confirm the importance of fine-tuned regulation of CREBdependent gene expression for CA1 neuron survival and function, and provide novel insight into the function of CREB in the etiology of neurodegenerative processes.

Cell Death and Differentiation (2010) 17, 1636-1644; doi:10.1038/cdd.2010.40; published online 16 April 2010

The cAMP-signaling pathway is activated in neurons in response to a wide array of stimuli. Synaptic activity, hormones, growth factors released during development, hypoxia, and stress, among other stimuli, can trigger the phosphorylation of the cAMP-response element-binding protein (CREB), causing its activation and the subsequent induction of a transient wave of CREB-dependent gene expression. ${ }^{1}$ This activity is necessary for the survival of different neuronal subtypes both in vitro and in vivo. ${ }^{2-6}$ The requirement for $C R E B$ is very stringent in the peripheral nervous system, ${ }^{7}$ whereas in the central nervous system most neuronal types are only affected when both CREB and the cAMP-response element modulator (CREM) are eliminated or inhibited. $^{8-10}$

Our earlier research on bitransgenic mice expressing either a strong dominant-negative CREB/CREM inhibitor (A-CREB) or a constitutively active CREB variant (VP16-CREB) revealed markedly different initial effects of repressing or boosting the activity of CREB in neuronal gene expression and physiology. A-CREB reduced intrinsic excitability and impaired long-term potentiation (LTP), ${ }^{10}$ whereas VP16CREB enhanced excitability ${ }^{11}$ and LTP. ${ }^{12}$ Strikingly, these divergent early effects led to apparently similar late deleterious effects, as both manipulations caused in the long term a severe loss of neurons in the CA1 subfield of the hippocampus in a dose- and time-dependent manner. ${ }^{10,11}$

Do both the blockade and the enhancement of CREB function trigger the same cell death program? Is CREB misregulation, as earlier proposed, ${ }^{13-18}$ involved in neurodegenerative diseases? To answer these questions, we performed parallel histological and cell death assays as well as gene profiling in the hippocampus of A-CREB and VP16CREB mice. We define the ultrastructural transcriptional signatures associated with these two forms of neurodegeneration, and show that a sustained blockade of CREB function leads to atrophy and dark neuron degeneration, whereas its strong chronic activation eventually causes excitotoxic cell death and widespread inflammation. These two processes are associated with differential activation of the immune response that determines the extent of the neuronal damage.

\section{Results}

Chronic inhibition of CREB function causes dark neuron degeneration. We used electron microscopy analysis to investigate the mechanism of cell death underlying the shrinkage of the CA1 subfield observed in brain sections of bitransgenic mice expressing the strong inhibitor of the CREB family of transcription factors A-CREB for several weeks. $^{10}$ The CA1 pyramidal cell layer of 6-week-old A-CREB mice, a time in which the loss of CA1 neurons started to become evident by Nissl staining, contained a large number of shrunken pyramidal cells with highly osmiophilic cytoplasm (Figure 1). These heteropycnotic cells, which represented up to $20 \%$ of the pyramidal neurons in the dorsal hippocampus of A-CREB mice (inset in Figure 1b), showed apparent nuclear and cytoplasmic condensation, clumping of chromatin, and their nuclear and plasma membranes were ruffled (Figure 1c). Although most subcellular organelles

\footnotetext{
${ }^{1}$ Instituto de Neurociencias de Alicante (Universidad Miguel Hernández-Consejo Superior de Investigaciones Científicas), Campus de Sant Joan, Apt. 18, Sant Joan d'Alacant, Alicante 03550, Spain and ²Departamento de Ciencias Médicas, Facultad de Medicina and Centro Regional de Investigaciones Biomédicas, Universidad Castilla-La Mancha, Campus Biosanitario, C/Almansa 14, Albacete 02006, Spain

*Corresponding authors: A Barco or LM Valor, Molecular Neurobiology, Instituto de Neurociencias de Alicante (UMH-CSIC), Campus de Sant Joan, Apt. 18, Sant Joan d'Alacant, Alicante 03550, Spain. Tel: + 34965919232 (AB), + 34965919531 (LMV); Fax: + 34965 919492; E-mail: abarco@umh.es or Imv@umh.es Keywords: cAMP pathway; neurodegeneration; excitotoxicity; apoptosis; inflammation Abbreviations: CREB, CAMP-response element-binding protein; CREM, CAMP-response element modulator; LTP, long-term potentiation Received 11.2.10; accepted 08.3.10; Edited by D Rubinztein; published online 16.4.10
} 

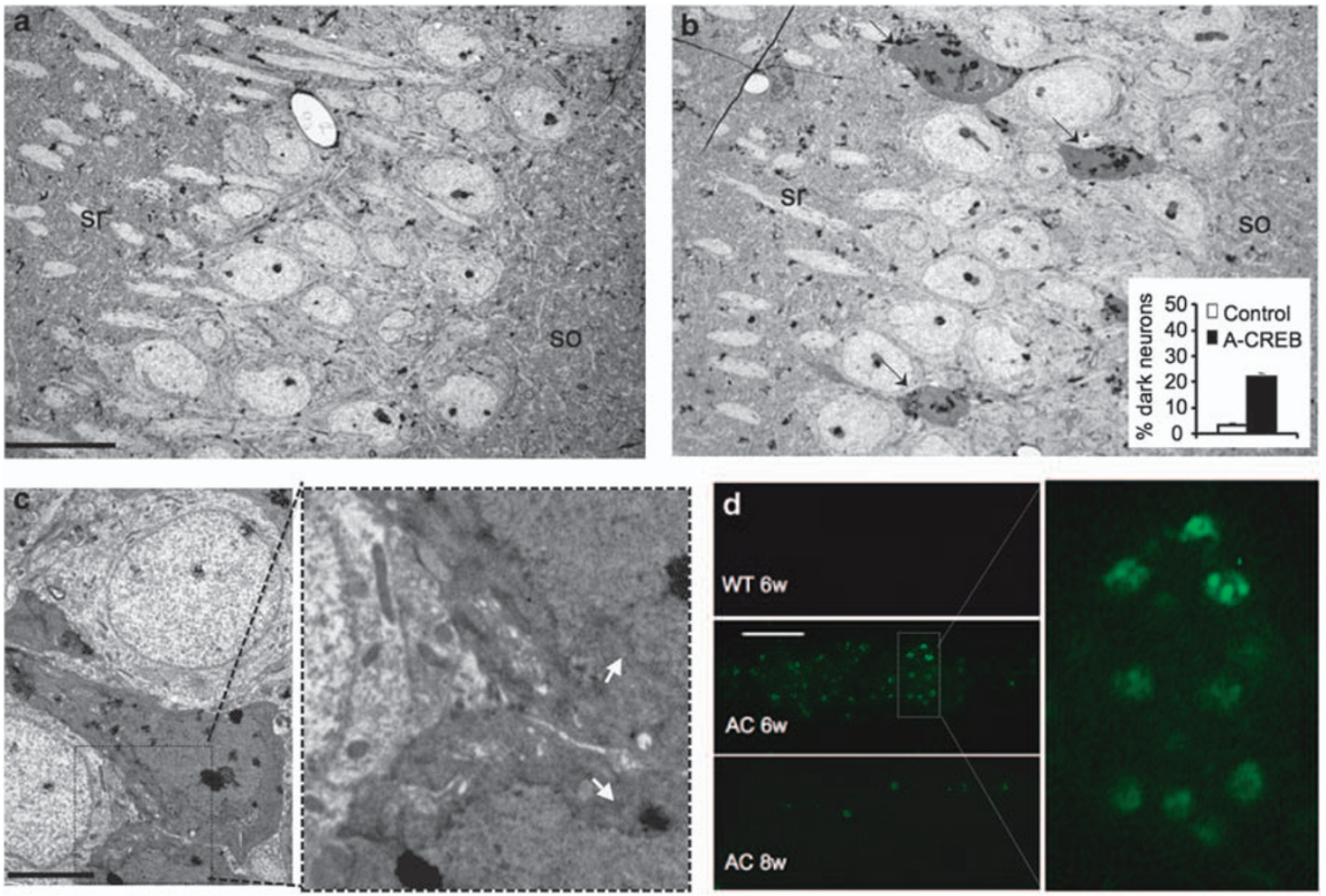

Figure 1 Death of A-CREB expressing neurons. Electron micrographs of pyramidal neurons in the CA1 subfield of the dorsal hippocampus of A-CREB mice. (a) Micrographs of the CA1 subfield in a control mouse. (b, c) Micrographs of the CA1 subfield in 6-week-old A-CREB mice reveal the presence of heteropycnotic pyramidal cells (black arrows in $\mathbf{b}$, quantification in bar graph inset) not observed in sections from control littermates. Heteropycnotic neurons showed early signs of apoptotic-like degeneration, consisting of cell bodies markedly reduced in size and increased electron density as a result of mild condensation of the nucleus and the cytoplasm, and morphologically injured mitochondria. The inset in (c) shows a higher magnification image of the convoluted nuclear membrane and the chromatin clumps around the inner membrane of the nucleus (white arrows). Scale: $A=20 \mu \mathrm{m} ; C=6 \mu \mathrm{m}$. (d) Representative images of TUNEL reaction in the hippocampus of 6- and 8-week-old A-CREB mice (AC) and control littermates (WT). Three control and four mutant mice (two per time point) were analyzed. Scale: $70 \mu \mathrm{m}$

seemed to be normal, some cells exhibited swelling of mitochondria and the Golgi network. There was no fragmentation of the nucleus or cytoplasm, and adjacent neurons had a normal appearance. This description fits with what has been referred to as dark cell or dark neuron degeneration, a form of neuronal death sometimes associated with positive TUNEL staining, a common marker of apoptotic cell death. ${ }^{19,20}$ In fact, the analysis of brain sections from A-CREB mice revealed the presence of TUNEL-positive neurons in the CA1 subfield preceding the spread of cell loss in this area (Figure 1d). At later times, when the thickness of the cellular layer was severely reduced and there were few cells positive for transgene expression, we did not observe cells positive for TUNEL staining (bottom panel). In agreement with these results, we also found neurons positive for active caspase-3, another marker of apoptotic cell death, in the sections from 6-week-old A-CREB mice (Supplementary Figure S1A). Brain sections of A-CREB mice were also positive for a reduced-silver staining that labels degenerating neurons (Supplementary Figure S1B). These results, together with the reduced excitability and impaired plasticity observed in earlier electrophysiological analyses, ${ }^{10}$ suggest that reduced responsiveness in A-CREB expressing neurons may lead to the activation of an apoptotic program that eliminates transgene expressing cells, explaining both the shrinkage of the cellular layer at the CA1 subfield and the loss of transgene expression in old mice.

\section{Chronic enhancement of CREB function causes} inflammation. Earlier studies have shown that bitransgenic mice expressing a constitutively active CREB variant at a high level (VP16-CREB ${ }^{\text {high }}$ ) showed enhanced intrinsic excitability and synaptic plasticity in the hippocampus shortly after transgene induction, ${ }^{11,12}$ but late spontaneous seizures and a loss of CA1 pyramidal neurons. ${ }^{11}$ In agreement with these results, the electron microscopy analysis of VP16$\mathrm{CREB}^{\text {high }}$ mice showed that the pyramidal cell layer maintained a normal appearance during the first 2 weeks after transgene induction (Figure $2 a$ and $\mathrm{f}-\mathrm{i}$ ). However, 3 weeks after transgene induction, incipient local signs of neurodegeneration were observed. Infiltrated glial cells, some cell debris, and vacuolated neurons were occasionally observed between the cell bodies of apparently intact CA1 pyramidal neurons (Figure $2 b$ and $d$ ). Six weeks after doxycyline (dox) removal, the number of pyramidal neurons was severely reduced and massive astrogliosis was apparent in both the cellular and the dendritic layer (Figure $2 \mathrm{c}$ and $\mathrm{e}$ ). The immunohistological analysis using antibodies against the astrocyte marker GFAP (Figure $3 a$ ) and the activated microglia marker $F 4 / 80$ 

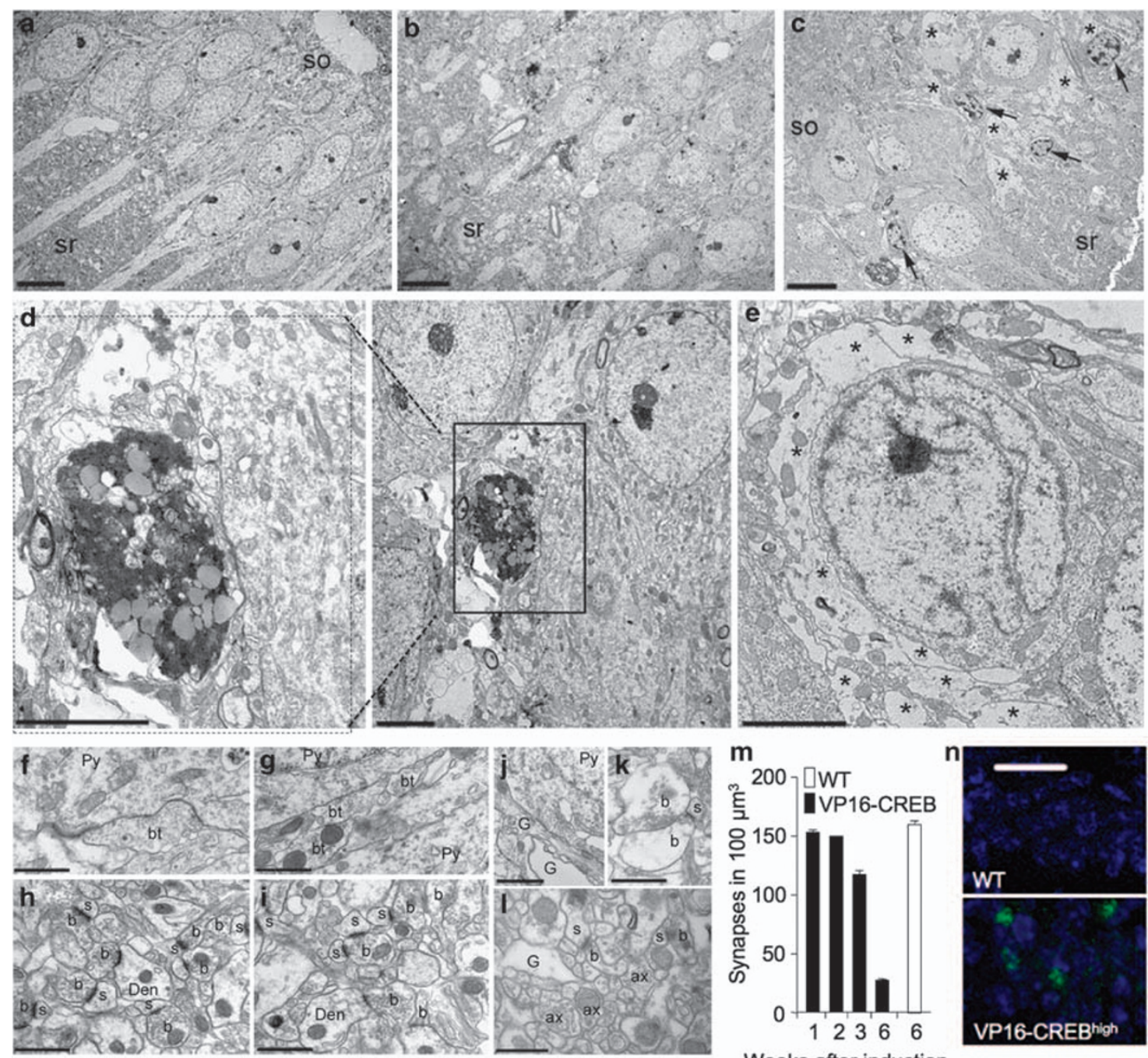

Figure 2 Death of VP16-CREB expressing neurons. (a) Electron micrographs of pyramidal neurons in the CA1 subfield of the dorsal hippocampus of VP16-CREB ${ }^{\text {high }}$ mice showing progressive neurodegeneration after transgene induction. Micrographs of the CA1 subfield in control littermates (a) were undistinguishable of those of VP16CREB $^{\text {high }} 1$ or 2 weeks after transgene induction. Three weeks after transgene induction, a number of ultrastructural changes were appreciable both in the cellular layer (b, $\mathbf{d}$; the left inset in $\mathbf{d}$ shows a higher magnification image of the framed area) and the stratum radiatum. Six weeks after transgene expression, the disorganization of the hippocampus was evident (c) and a large number of glial cells were found in the stratum radiatum and in the proximity of pyramidal cell bodies; in some cases, phagocytosing those cells (e). Abbreviations: sr, stratum radiatum; so, stratum oriens. Asterisk, glial cell process; black arrow, glial cell nucleus. Scale bars: $(\mathbf{a}-\mathbf{c})=5 \mu \mathrm{m} ;(\mathbf{d}-\mathbf{e})=2 \mu \mathrm{m}$. $(\mathbf{f}-\mathbf{l})$ Electron microscopy of synapses in the stratum radiatum of VP16-CREB mice. Micrographs in control littermates $(\mathbf{f}, \mathbf{h})$ were undistinguishable of those of VP16-CREB ${ }^{\text {high }}$ one (results not shown) or 2 weeks after transgene induction $(\mathbf{g}$, i). Both inhibitory (f, $\mathbf{g})$ and excitatory synapses (h, i) were normal. 3 weeks after transgene induction, a number of ultrastructural changes started to be appreciable in both inhibitory (j) and excitatory (k) synapses. Six weeks after transgene expression (I), the disorganization of the stratum radiatum was profound. Abbreviations: Py, pyramidal cell; bt, basket cell terminal; s, spine; b, button or axon terminal; Den, dendritic shaft; ax, axons; G, glial cell process. Scale bars: $0.5 \mu \mathrm{m}$. $(\mathrm{m})$ Quantification of the number of excitatory synapses at different time points after transgene induction. (n) Representative images of TUNEL reaction (green) in the hippocampus of VP16-CREB ${ }^{\text {high }}$ mice and control littermates 3 weeks after transgene induction $(n=3$ for both genotypes). Sections were counterstained with DAPI (blue). Scale: $50 \mu \mathrm{m}$

(Figure $3 b$ ) confirmed the active gliosis observed in the electron microscopy images.

In terms of the number and morphology of synapses in the stratum radiatum, we did not observe significant differences 2 weeks after induction in either inhibitory or excitatory synapses (Figure 2f-i). However, there was a pronounced reduction of glutamatergic synapses in mice expressing the transgene for 6 weeks (Figure $2 \mathrm{~m}$ - wt: $166 \pm 4 \mathrm{syn} / 100 \mathrm{~mm}^{3}$; VP16-CREB $\left.{ }^{\text {high: }} 27 \pm 2 \mathrm{syn} / 100 \mathrm{~mm}^{3} ; P<0.001\right)$. At these late times, we also observed ultrastructural abnormalities both at postsynaptic and presynaptic sites. Dendritic spines seemed smaller and elongated, whereas axonal terminals were enlarged and contained fewer synaptic vesicles and mitochondria (Figure 2j-l).

Although it is now believed that seizure-induced neuronal death is primarily morphologically necrotic, excitotoxic insults result in mixed apoptotic-necrotic features depending on their intensity. ${ }^{20,21}$ Consistent with this view, we found cells positive for TUNEL reaction in the hippocampus of VP16CREB $^{\text {high }}$ mice 3 weeks after induction (Figure 2n). Brain sections obtained at either earlier (1-2 weeks) or later (6 or more weeks) times after transgene induction were negative 


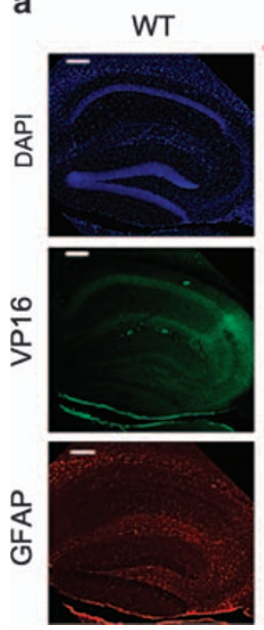

VP16-CREBhigh
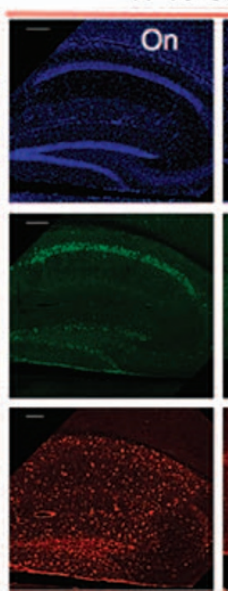
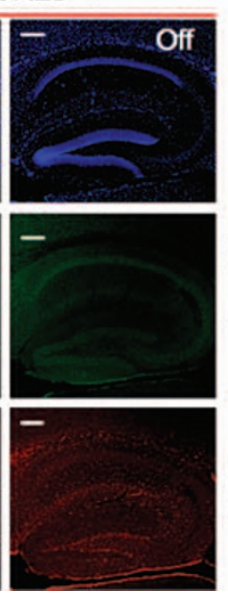

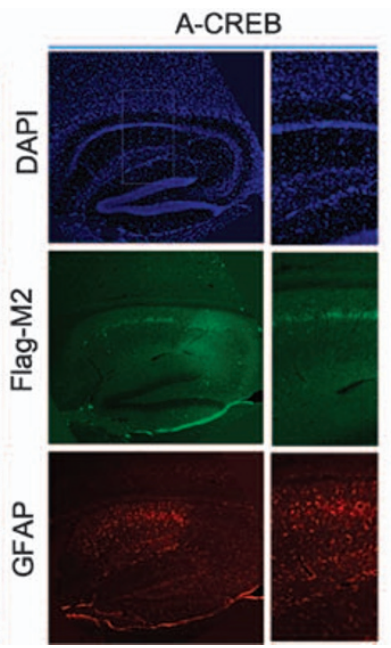

b

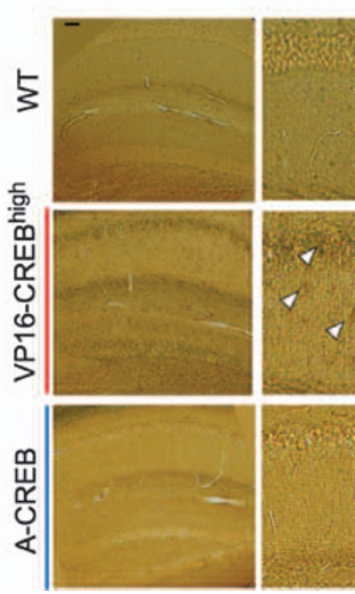

Figure 3 Gliosis in VP16-CREB ${ }^{\text {high }}$ mice. (a) Left, GFAP immunostaining of the hippocampus of VP16-CREB ${ }^{\text {high }}$ mice expressing (On, 3 weeks after transgene induction) or not expressing the transgene (Off, animals fed with dox food). Sections were double stained with antibodies against GFAP (red) and VP16 (green) and counterstained with DAPI (blue). Right, GFAP immunostaining of the hippocampus of 6-week-old A-CREB mice. Sections were double stained with antibodies against GFAP (red) and Flag-M2, which recognizes A-CREB (green), and counterstained with DAPI (blue). The right insets show higher magnification images. At least three independent mice per condition were used in this analysis. (b) Immunostaining using antibodies against the microglia marker F4/80 revealed the presence of reactive microglia only in the hippocampus of VP16-CREB ${ }^{\text {high }}$ mice (white arrows in higher magnification insets), whereas A-CREB mice did not show enhanced signal for this marker. Three bitransgenic mice and three control littermates were used in this comparison (the upper panel corresponds to a VP16-CREB control littermate). Scale bars: $140 \mu \mathrm{m}$

for this assay. The brain sections of VP16-CREB ${ }^{\text {high }}$ mice were also positive for silver staining, (Supplementary Figure S1B). Neither cell loss nor TUNEL or neurosilver-positive neurons were observed in bitransgenic mice that express lower levels of VP16-CREB protein (VP16-CREB ${ }^{\text {low }}$ ), even after 1 year of transgene expression (results not shown).

The features of degenerating neurons in VP16-CREB ${ }^{\text {high }}$ mice, together with the enhanced excitability of VP16-CREB expressing neurons and the spontaneous epileptic activity, ${ }^{11}$ indicate that cellular loss in the hippocampus of these mice is primarily caused by excitotoxic neuronal death and includes degenerating neurons undergoing apoptotic cell death. Notably, the inflammatory process associated with strong chronic activation of the CREB pathway was absent or very minor in mice with reduced CREB function: GFAP immunoreactivity was stronger and more widespread in the hippocampus of VP16-CREB ${ }^{\text {high }}$ mice than in A-CREB mice (Figure $3 a$ ), and reactive microglia was detected only in the former (Figure 3b). These observations were later confirmed by gene expression analysis (Figure 4g; Supplementary Figure S3; Supplementary Tables 1 and 2).

Gene expression profiles of chronic inhibition or enhancement of CREB function. To gain additional insight into the pathological processes triggered by abnormal activity in the CREB pathway, we investigated the gene expression profiles active during ongoing neurodegeneration using microarrays. RNA samples from bitransgenic mice at the onset of neurodegeneration (i.e. 6week-old A-CREB mice and VP16-CREB ${ }^{\text {high }}$ mice 3 weeks after transgene induction, referred to as A-CREB and VP16CREB late samples, respectively) were compared with those from their respective control littermates. For comparison purposes, we also examined samples obtained at earlier time points of transgene expression, in which there were no evident signs of neuronal death (3-week-old A-CREB mice and VP16-CREB ${ }^{\text {high }}$ mice 1 week after transgene induction, referred as A-CREB early and VP16-CREB early, respectively).

As expected for an activity-regulated transcription factor, CREB inhibition had a milder effect on basal gene expression than its overactivation both at early (51 versus 280 probe sets) and late times (62 versus 1566 probe sets) of transgene expression (Figure 4a; Supplementary Tables 1 and 2). We found that at early times gene downregulations prevailed, although not significantly, in A-CREB mice $(60.8 \%, P=0.32$, Fisher's exact test), whereas upregulations were more frequent in VP16-CREB mice $\left(85.4 \%, P=1.66 \times 10^{-19}\right)$. To some extent, these numbers are in agreement with the expected effect of a dominant negative and a constitutively active form of a transcription factor. In contrast, at later times, we observed a dramatic increase in the number of upregulated probe sets in A-CREB mice $(39.2 \%$ in early samples compared with $95.2 \%$ in late samples, $P=4 \times 10^{-11}$ ) and the activation of a broad genetic program ( $>1000$ genes) in VP16-CREB mice that included a large number of downregulated genes (41 probe sets in early samples compared with 336 probe sets in late samples, $P=0.001$ ). The differential profile of gene expression at early and late times in both mutant strains might be caused by the activation of secondary genetic programs and/or changes in the cellular composition of the hippocampus. Thus, in the case of VP16CREB, we found an enrichment of CREB-binding sites in the promoter of upregulated genes at early times after transgene induction (Pscan $z$-test $P$-value: 0.01 ; see Supplementary Methods), but not at later time $(P=0.13)$. Interestingly, $\sim 74 \%$ of the probe sets significantly altered in the A-CREB late samples were also affected in the VP16-CREB late samples (Figure $4 \mathrm{~b}$ and $\mathrm{c}$ ). This common list, largely made of upregulated probe sets ( $\sim 96 \%)$, revealed changes that were in the same direction in A-CREB and VP16-CREB mice, 


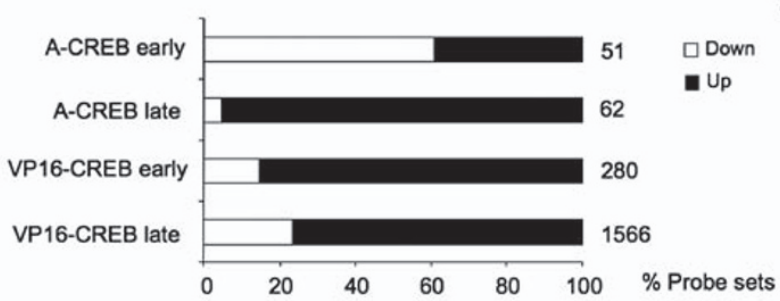

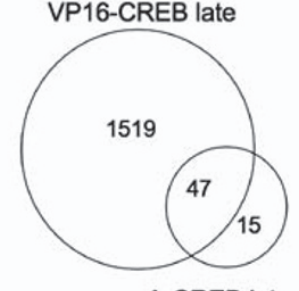

b

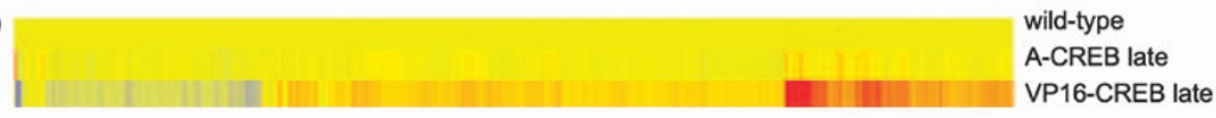

d

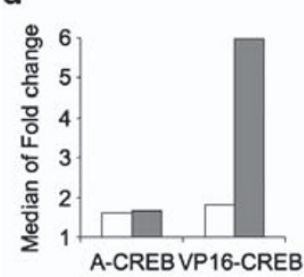

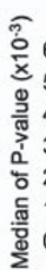

\section{e}

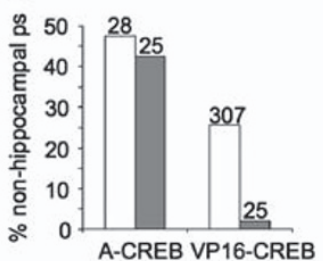

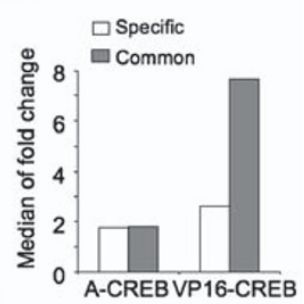

f

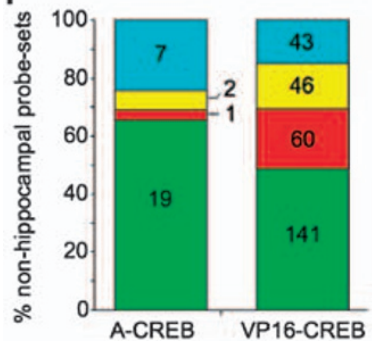

g
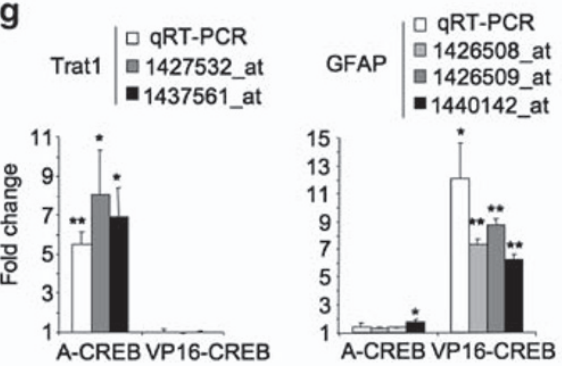

\begin{tabular}{l|l} 
H2-Q7 & $\begin{array}{l}\text { qRT-PCR } \\
\text { a 1418536_at }\end{array}$
\end{tabular}

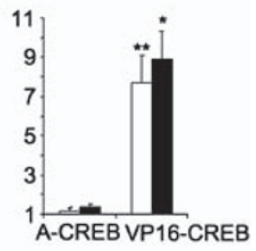

Figure 4 Comparison of gene expression profiles in the hippocampus of A-CREB and VP16-CREB ${ }^{\text {high }}$ mice. (a) The percentage of upregulated (black) and downregulated (white) probe sets at early and late times, as well as the number of probe sets altered in each condition (number on the right side), is shown. (b) Squared-Euclidean hierarchical clustering of the significantly altered probe sets in both bitransgenic hippocampi. Red, upregulation; yellow, no change; blue, downregulation. (c) Venn diagram presenting the total number of affected probe sets and the overlap in the profiles of A-CREB and VP16-CREB late samples. (d) Graphs showing the median of the fold changes (left) as a measure of the magnitude of the changes, and the median of the $P$-values (right) as a measure of their significance. 'Specific' (white bars) refers to probe sets that passed the filters in only one of the two bitransgenic strains, whereas 'Common' (gray bars) passed in both strains. (e) Percentage of non-hippocampal probe sets (left) and median of the fold change (right) in either A-CREB or VP16-CREB high late samples (white bars), and common to both strains (gray bars). The number of probe sets is indicated above each bar. (f) Percentage of non-hippocampal genes and associated cell types: macrophages and microglia (green), dendritic and mast cells (red), glial cells (yellow), and others (blue). The numbers represent the number of genes for each cell group (see Supplementary Figure 3 for additional details). (g) qRT-PCR assays for selected genes and comparison with the fold change of the corresponding probe sets in the microarray analysis. ${ }^{*} P<0.05$; ${ }^{* *} P<0.01$ Student's $t$-test

although their magnitude and statistical significance were consistently larger in VP16-CREB mice (Figure 4d).

Differential immune response in CREB mutant strains. Enrichment analysis for gene ontology (GO) terms showed that genes related with the immune response (complement proteins, chemokines, etc.) were overrepresented among the upregulated genes in both mutant strains (Supplementary Figure S2). The GO terms antigen presentation (containing both $\mathrm{MHC}$ class I and II), production of cytokines, interferon and tumor necrosis factor, and wounding were specifically associated with the VP16-CREB late samples. Owing to the prominent upregulation of nonneuronal markers related with the immune response in the gene expression profiles of both mutant strains, we evaluated in greater detail the presence of infiltrated immune cells in hippocampal tissue by taking advantage of the publicly available gene expression profiles of several types of immune and glial cells (see Supplementary Methods). More than $47 \%$ of the significantly altered probe sets in the A-CREB late samples seemed to have a nonhippocampal origin, and the majority of them were also upregulated in the VP16-CREB ${ }^{\text {high }}$ late samples (Figure 4e). Although these probe sets represented a small proportion of the non-hippocampal probe sets in the VP16-CREB late $(\sim 2 \%)$, they ranked among the most prominently changing probe sets (median of the fold change $>7$ ). Our metaanalysis indicated that most of the non-hippocampal probe sets in the A-CREB late samples could be explained by the presence of macrophage-related cells (Figure 4f; Supplementary Figure S3A). Interestingly, this analysis revealed three probe sets, corresponding to the T-cell receptor-associated transmembrane adapter 1 (Trat1) and activated leukocyte cell adhesion molecule (Alcam) genes, that were strongly upregulated in A-CREB samples, but unaltered in VP16-CREB late samples (see Discussion). In 
contrast, the presence of non-hippocampal probe sets at late times in the hippocampus of VP16-CREB mice is better explained considering the summation of the molecular signatures of different immune cell types (Figure 4f; Supplementary Figure S3B). This suggests that a more extensive and specialized immune response and stronger gliosis take place in the hippocampus of VP16-CREB ${ }^{\text {high }}$ mice than in that of A-CREB mice. Both histological analyses presented above and qRT-PCR assays for selected genes (Figure $4 \mathrm{~g}$ ) are in good agreement with these observations.

Comparison with other neurodegenerative processes. To gain new insight into the function of CREB in neurodegenerative processes, we compared the gene expression profiles observed in CREB mutants with those associated with different neurodegenerative conditions in which the deregulation of CREB activity had been earlier documented or proposed. This is the case of Huntington's disease (HD), ${ }^{13,14,22}$ Alzheimer's disease (AD), ${ }^{15,23}$ epilepsy, ${ }^{16,24}$ and aging. ${ }^{17,25-27}$ On the basis of some obvious similarities with a recently published gene profiling analysis, ${ }^{28}$ we extended our comparison with the knockout mouse for palmitoyl-protein thioesterase 1 (PPT1), an animal model for infantile Batten's disease (BD). This inheritable disorder, which has not been earlier associated with CREB misregulation, is characterized by epilepsy and retinal and cortical neurodegeneration. ${ }^{29}$

This meta-analyses revealed limited overlap between the gene profiles of CREB mutants and those of $A D$ and $H D$ mouse models (Figure 5a). The few genes in common between the $A D$ mouse model and the CREB animals were functionally related with inflammatory responses (Supplementary Table 3), whereas in the case of HD mouse models, common changes were primarily associated with the downregulation of neuronal genes, including some potential direct targets of CREB, such as c-fos or Prodynorphin (Supplementary Table 3 and results not shown). The profile of VP16CREB-mediated neurodegeneration showed strong correlations with infantile BD and the kainate model of epilepsy (Figure 5a; Supplementary Figure S4A-C), which encourage further examination of the function of CREB in these pathologies. In contrast, A-CREB profile exhibited very little overlapping with other neurodegenerative profiles, suggesting that dark cell neurodegeneration is rare in those pathological conditions, whereas inflammation was much more prevalent. Interestingly, the inspection of the neurodegenerative markers common to several conditions revealed two significantly enriched pathways $(P<0.05)$, which correspond to initial steps in the immune responses: the activation of the complement and coagulation cascades, and antigen processing and presentation (Supplementary Figure S4D). These pathways were overrepresented in nearly all the tested conditions, highlighting their relevance in neurodegenerative disorders (Figure 5b; Supplementary Table 3).

\section{Discussion}

The picture that emerges from the interdisciplinary analysis of CREB mutants with a loss or a gain of function together with earlier studies in CREB-deficient mice indicates that the
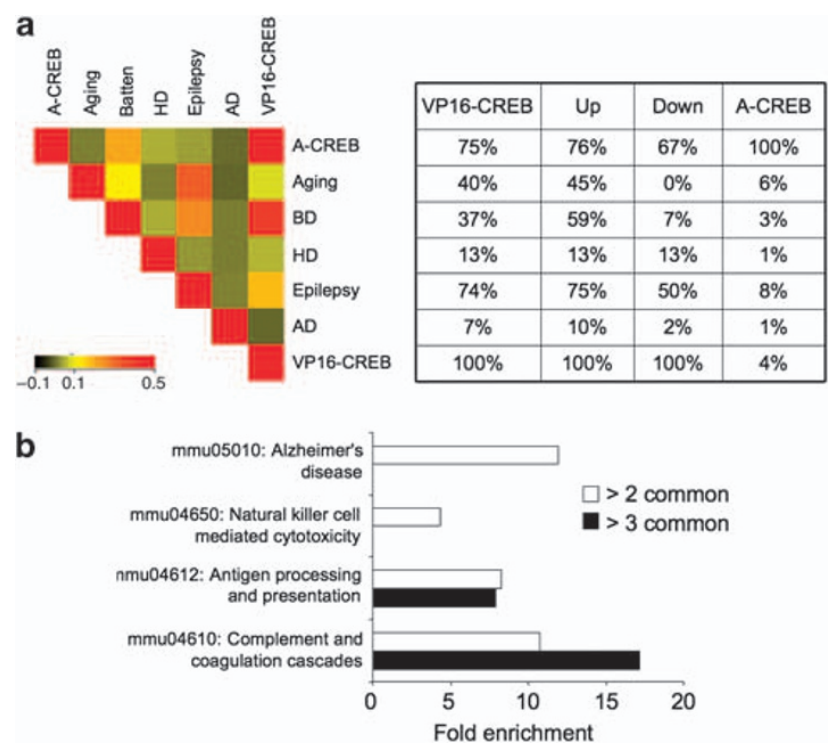

Figure 5 Meta-analysis of neuropathological models. (a) Correlation analysis of the transcriptional alterations associated with the following pathological conditions: mouse model of $A D$ (Presenilin conditional double knockout), aging (24 months old animals), kainate-induced epilepsy (10 days after injection), $H D$ (R6/1, R6/2, and CHL2), BD (PPT1 8 months old knockout), and A-CREB and VP16-CREB high mice at late times after transgene expression. See coloring legend for coefficient values. The percentage of altered genes common to the late expression profiles of VP16-CREB ${ }^{\text {high }}$ mice and A-CREB mice is indicated in the right table. 'Up' and 'Down' distinguish between upregulated and downregulated genes in VP16-CREB samples, respectively. (b) Significative enrichment for canonical KEGG pathways reveals the presence of complement and antigen presentation genes in the profiles of neurodegenerative models. Analysis was performed using genes present at least in three ( $>2$ common, white bars) or four ( $>3$ common, black bars) ( $P$-value $<0.05$, see Supplementary Methods)

precise temporal control of CREB-dependent gene expression is a critical requirement for the viability of CA1 pyramidal neurons and that any imbalance in the activity of this transduction cascade can have a negative impact in the survival and function of these cells, likely through misregulation of their responsiveness to external stimuli, as the electrophysiological alterations preceded the onset of neurodegeneration.

Here, we presented first the ultrastructural signature associated with neurodegeneration induced by chronic inhibition or activation of CREB function. Degenerating neurons in A-CREB mice characteristically exhibited intracellular inclusions, condensation of both the cytoplasm and the nucleus, and ruffling of the plasma membrane, whereas the ultrastructure of cellular organelles was largely preserved. There was no inflammatory response accompanying neurodegeneration, and as in classical apoptosis, there was minimal damage in the surrounding cells. In contrast, in VP16-CREB mice, we observed a strong activation of astroglia and microglia as well as other ultrastructural features that suggested an excitotoxic death, in which cellular contents are liberated into the intercellular space damaging neighboring neurons and inducing an inflammatory response. In agreement with these scenarios, the gene profiling analysis showed that the number of markers for inflammation and the magnitude and significance of the changes were higher in 
CHRONIC ACTIVATION

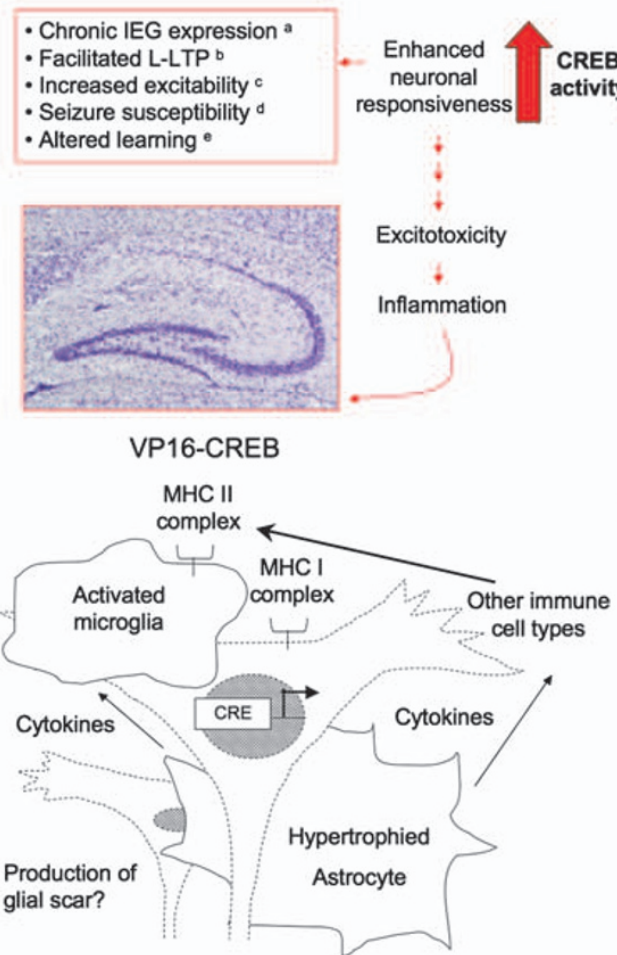

CHRONIC INHIBITION

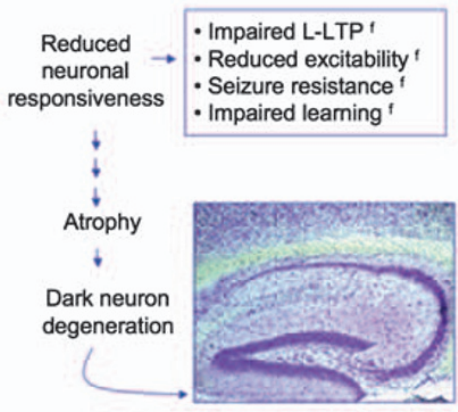

\section{A-CREB}

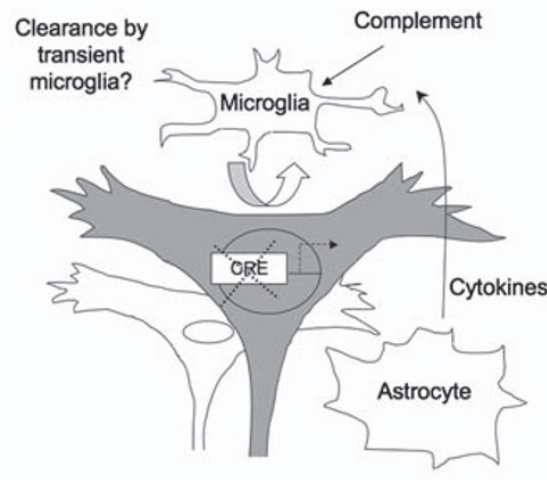

Figure 6 Bidirectional control of CA1 neuronal survival and plasticity by CREB. Summary of opposite and coincidental alterations detected during the multidisciplinary characterization of transgenic strains with regulatable enhancement or inhibition of CREB function. The bottom drawings show heuristic models for the cell death mechanisms activated in each mouse strain according to the results of the ultrastructural and transcriptional profiling. ${ }^{a}$ Barco et al. ${ }^{12}$, Barco et al. ${ }^{33}$; barco et al. ${ }^{12}$ and others; ${ }^{\circ}$ Lopez de Armentia et al. ${ }^{11}$ and others; ${ }^{d}$ Jancic et al. ${ }^{10}$, Lopez et al. ${ }^{11}$; ${ }^{\text {VViosca }}$ et al. ${ }^{40}$ and others; ${ }^{\dagger}$ Jancic et al. ${ }^{10}$ and others

VP16-CREB mice than in A-CREB mice and provided interesting cues about the differential evolution of both neurodegenerative processes.

In A-CREB mice, the expression of immune-response genes was restricted to some chemokines and components of the complement and phagocytic systems. Interestingly, two immune genes, Alcam and Trat1 (T-cell receptor interacting molecule or Trim), were strongly upregulated in A-CREB mice and might act as negative modulators of the immune response. Alcam inhibits the transendothelial migration of monocytes, ${ }^{30}$ thus providing a possible mechanism for limiting the infiltration of immune cells from brain capillaries, whereas Trat1 regulates the surface transport of CTLA-4, an immunoglobulin receptor that dampens T-cell responses (reviewed in Rudd et al. ${ }^{31}$ ). Therefore, the increase of Trat1 expression in microglia might limit its activation. These molecules could functionally interact with other negative modulators such as the anti-inflammatory Serping1, also called $\mathrm{C} 1$ inhibitor, and the autoimmunity suppressor Fc- $\gamma$ IIB (Fcgr2b), whose expression is also increased in the hippocampus of A-CREB mice (Supplementary Table 1) and could refrain the full activation of the complement pathway and prevent inflammation. In the absence of an inflammatory response, the number of microglia cells might be so low or transient that it escaped our microscopy analysis, but sufficient to increase the expression of innate immune-response markers detected in the gene expression profile, and to clear out apoptotic cells without damaging the surrounding neurons ${ }^{32}$ (Figure 6, right).
In the hippocampus of VP16-CREB ${ }^{\text {high }}$ mice, Alcam and Trat1 were not upregulated and the strong activation of microglia (evidenced by the F4/80 marker and the strong upregulation of Emr1) and the complement cascade correlated with a more dramatic cellular response. The overexpression of $\mathrm{MHC}$ class I molecules in pyramidal neurons of VP16-CREB ${ }^{\text {high }}$ mice, ${ }^{33}$ maybe physiological in origin, ${ }^{34}$ might convert them into antigen-presenting cells, fuelling the inflammatory process. As a result, we observed glial overactivation (evidenced by the increase in GFAP, aquaporin-4, and other glial markers), the infiltration of peripheral immune cells such as T- and B-lymphocytes and dendritic cells, and the release of pro-inflammatory molecules (MHC class II molecules, interleukines, etc.) that may work in a positive feed-forward manner (Figure 6, left).

Our characterization of CREB mutant strains suggests that the strength of complement activation could determine the magnitude and features of the immune response associated with a neurodegenerative process. The two bitransgenic lines described here illustrate two different faces of the immune response in the nervous system: full inflammatory response versus ordered cell death clearance. This view is supported by the EM analysis, the cell death assays, and the microarray gene profiling. However, the precise identity of the molecular determinants that separate the function of the complement system in focalized cellular clearance from aggressive inflammation remains undetermined. ${ }^{35}$ Further use of the analytical approaches described here should define distinctive 
transcriptional signatures and provide additional clues about the molecular and cellular mechanisms underlying neurodegeneration and maybe reveal novel therapeutic targets.

Finally, these results highlight the perils of prolonged and/or uncontrolled manipulation of the CREB-signaling cascade. Although CREB activity has been shown to be neuroprotective in several models of neuronal damage, ${ }^{4,6,36-39}$ it is essential to be careful with the duration and strength of CREBbased therapeutics. In the healthy brain, the phosphorylation of CREB that occurs in response to neuronal activity is transient and promotes the expression of specific downstream genes in a narrow time window. The blockade of this function has dramatic effects in neuronal viability, but the maintenance of strongly elevated levels of CREB activity can be equally devastating.

\section{Materials and Methods}

Generation and maintenance of transgenic mice. A-CREB ${ }^{10}$ and VP16-CREB ${ }^{\text {high } 12}$ mice have been described earlier. Both strains have been backcrossed to C57BL/6J for over 20 generations. In all our experiments, we used littermate mice carrying either no transgene or the ITA or tetO transgene alone as controls. For VP16-CREB ${ }^{\text {high }}$ mice and control littermates, dox was administrated at $40 \mathrm{mg} / \mathrm{kg}$ of food and removed to induce transgene expression at the indicated times before experimentation. A-CREB mice were raised without dox. Animal procedures were performed according to the $\mathrm{EU}$ and national guidelines for the use of laboratory animals.

Histological techniques. Immunohistochemistry stainings were performed as earlier described. ${ }^{11}$ The following primary antibodies were used: $\alpha$-cleaved caspase-3 antibodies (Cell Signalling Technology, Beverly, MA, USA), $\alpha$-VP16 (Santa Cruz Biotechnology Inc., Santa Cruz, CA, USA), $\alpha$-Flag-M2, $\alpha$-F4/80, and $\alpha$-GFAP (Sigma Aldrich Química S.A., Madrid, Spain). Secondary biotinylated antibodies, streptividin-peroxidase conjugate, and the DAB substrate were obtained from Sigma and fluorescent secondary antibodies from Invitrogen (Carlsbad, CA, USA). For TUNEL reaction, serial brain sagittal cryosections of $12-\mu \mathrm{m}$ thickness were stained using the in situ Cell Death Detection kit (Roche Applied Science, Barcelona, Spain). Silver staining was carried out using the FD Neurosilver kit (FD NeuroTechnologies Inc., Catonsville, MD, USA).

Electron microscopy. For electron microscopy, mice were anesthetized and perfused with $4 \%$ paraformaldehyde and $1 \%$ glutaraldehyde in $0.1 \mathrm{M} \mathrm{PB}(\mathrm{pH} 7.4)$. Then, coronal sections were cut with a thickness of $60 \mu \mathrm{m}$ at the level of the dorsal hippocampus using a Leica vibration microtome. After several washes in PB, the sections were postfixed with osmium tetraoxide ( $1 \%$ in $0.1 \mathrm{M} \mathrm{PB}$ ) and block stained with uranyl acetate ( $1 \%$ in distilled water). The sections were then dehydrated in ascending series of ethanol to $100 \%$ followed by propylene oxide and flat embedded on glass slides in Durcupan (Fluka, Barcelona, Spain). The CA1 region of the hippocampus was cut at $70-90 \mathrm{~nm}$ on an ultramicrotome (Reichert Ultracut $\mathrm{E}$; Leica, Austria) and collected on 200-mesh nickel grids. Staining was performed on drops of $1 \%$ aqueous uranyl acetate followed by Reynolds's lead citrate. Ultrastructural analyses were performed with a Jeol-1010 electron microscope.

Quantitative RT-PCR. qRT-PCR was carried out in an Applied Biosystems 7300 real-time PCR unit using SYBR GreenER mix (Invitrogen) and primers specific for Trat1, glial fibrillary acidic protein (GFAP), the major histocompatibility component H2-Q7 and glyceraldehyde 3-phosphate dehydrogenase (GAPDH). The sequences are as follow: Trat1 sense, $5^{\prime}$-CACAGGTACCGGCTGAAGCT-3'; Trat1 antisense, 5'-CGCTGTGATCCAGTGAGGC-3'; GFAP sense, 5'-GGACAAC TTTGCACAGGACCTC-3'; GFAP antisense, 5'-TCCAAATCCACACGAGCCA-3'; H2-Q7 sense, $5^{\prime}$-AGGAGCAGAATTACACATGCCA; H2-Q7 antisense, 5'-CGCCA TGTTGGAGACAGTGTAT, GAPDH sense, $5^{\prime}$-CTTCACCACCATGGAGAAGGC-3'; and GAPDH antisense, $5^{\prime}$-CATGGACTGTGGTCATGAGCC-3'. Each independent sample was assayed in duplicate and normalized using GAPDH levels.

Microarray analysis. For each mouse genome 4302.0 gene expression array (Affymetrix, Santa Clara, CA, USA), total RNA was extracted from the hippocampi of three to four mice with the same age, sex, and genotype to produce one pooled sample. We analyzed three late A-CREB pooled samples (6-week-old mice) and three late VP16-CREB pooled samples (3-week-off dox) with their corresponding control littermate samples (three pooled samples for each strain). To compare with early changes, we included two pooled A-CREB early samples (3-week-old mice) and two pooled VP16-CREB early samples (1-week-off dox) with their corresponding control littermate samples. In the case of A-CREB mice, we used the dataset GSE14320. ${ }^{10}$ However, in the case of VP16-CREB mice, as earlier gene expression analyses ${ }^{33}$ were performed with old MG-U74v2 setA arrays that only interrogated a third of the genome, we prepared new samples from bitransgenic mice and control littermates 1 week after transgene induction and hybridized them to mouse genome 4302.0 genechips. The new datasets generated in this study can be found at the gene expression omnibus (GEO) database with the accession number GSE21137. The arrays were hybridized, washed, and screened for quality according to the manufacturer's protocol. The microarray data was then analyzed using GeneSpring GX 10.0 (Agilent Technologies, Inc., Santa Clara, CA, USA). An RMA algorithm was used for data extraction. Principal component analysis revealed clustering of samples according to the batch of replicates; therefore, normalization was conducted using the corresponding control samples values as reference. The filtering criteria were signal intensities $>20 \%$ of the maximal expression in all the samples in at least one condition, a fold change $>1.5$, and $P<0.05$ in the unpaired Student's $t$-test. The hierarchical clustering was also performed using GeneSpring software. Additional bioinformatics analyses and the meta-analyses to examine the presence of immune and glial cells markers in the gene expression profiles of CREB mutants and to compare with other neurodegenerative conditions are described in Supplementary Methods.

\section{Conflict of Interest}

The authors declare no conflict of interest.

Acknowledgements. We thank Eva Benito, Eloisa Herrera, Frederic Laumonnier, and Jose P Lopez-Atalaya for critical reading of the manuscript and Roman Olivares for excellent technical assistance. The assistance of Eva Serna and members of the Unidad de Análisis Multigénico at the Facultad de Medicina in the Universidad de Valencia is also acknowledged. The work at AB and LMV laboratory was supported by the European Commission Coordination Action ENINET (contract number LSHM-CT-2005-19063), the Spanish Ministry of Science and Innovation grants BFU2005-00286, CSD2007-00023, and SAF2008-00611, the Generalitat Valenciana grant GVPRE/2008/365, and the Fundació La Marató de TV3 grant 063510. The work at RL laboratory was supported by the Spanish Ministry of Science and Innovation grant BFU2006-01896 and the Junta de Comunidades de Castilla-La Mancha grant PAI08-0174-6967.

1. Johannessen M, Delghandi MP, Moens U. What turns CREB on? Cell Signal 2004; 16 : 1211-1227.

2. Riccio A, Ahn S, Davenport CM, Blendy JA, Ginty DD. Mediation by a CREB family transcription factor of NGF-dependent survival of sympathetic neurons. Science 1999; 286 : 2358-2361.

3. Papadia S, Stevenson P, Hardingham NR, Bading H, Hardingham GE. Nuclear Ca2+ and the CAMP response element-binding protein family mediate a late phase of activitydependent neuroprotection. J Neurosci 2005; 25: 4279-4287.

4. Andreatta CP, Nahreini P, Hanson AJ, Prasad KN. Regulated expression of VP16CREB in neuroblastoma cells: analysis of differentiation and apoptosis. J Neurosci Res 2004; 78: 570-579.

5. Glover CP, Heywood DJ, Bienemann AS, Deuschle U, Kew JN, Uney JB. Adenoviral expression of CREB protects neurons from apoptotic and excitotoxic stress. Neuroreport 2004; 15: 1171-1175

6. Lee B, Butcher GQ, Hoyt KR, Impey S, Obrietan K. Activity-dependent neuroprotection and CAMP response element-binding protein (CREB): kinase coupling, stimulus intensity, and temporal regulation of CREB phosphorylation at serine 133. J Neurosci 2005; 25: 1137-1148

7. Lonze BE, Riccio A, Cohen S, Ginty DD. Apoptosis, axonal growth defects, and degeneration of peripheral neurons in mice lacking CREB. Neuron 2002; 34: 371-385.

8. Parlato R, Rieker C, Turiault M, Tronche F, Schutz G. Survival of DA neurons is independent of CREM upregulation in absence of CREB. Genesis 2006; 44: 454-464.

9. Mantamadiotis T, Lemberger T, Bleckmann SC, Kern H, Kretz O, Martin Villalba A et al. Disruption of CREB function in brain leads to neurodegeneration. Nat Genet 2002; 31: 47-54. 
10. Jancic D, Lopez de Armentia M, Valor LM, Olivares R, Barco A. Inhibition of CAMP response element-binding protein reduces neuronal excitability and plasticity, and triggers neurodegeneration. Cereb Cortex 2009; 19: 2535-2547.

11. Lopez de Armentia M, Jancic D, Olivares R, Alarcon JM, Kandel ER, Barco A. cAMP response element-binding protein-mediated gene expression increases the intrinsic excitability of CA1 pyramidal neurons. J Neurosci 2007; 27: 13909-13918.

12. Barco A, Alarcon JM, Kandel ER. Expression of constitutively active CREB protein facilitates the late phase of long-term potentiation by enhancing synaptic capture. Cell 2002; 108: 689-703

13. Shimohata T, Nakajima T, Yamada M, Uchida C, Onodera O, Naruse S et al. Expanded polyglutamine stretches interact with TAFII130, interfering with CREB-dependent transcription. Nat Genet 2000; 26: 29-36.

14. Sugars KL, Brown R, Cook LJ, Swartz J, Rubinsztein DC. Decreased cAMP response element-mediated transcription: an early event in exon 1 and full-length cell models of Huntington's disease that contributes to polyglutamine pathogenesis. J Biol Chem 2004; 279: 4988-4999

15. Vitolo OV, Sant'Angelo A, Costanzo V, Battaglia F, Arancio O, Shelanski M. Amyloid beta -peptide inhibition of the PKA/CREB pathway and long-term potentiation: reversibility by drugs that enhance CAMP signaling. Proc Natl Acad Sci USA 2002; 99 : 13217-13221.

16. Park SA, Kim TS, Choi KS, Park HJ, Heo K, Lee BI. Chronic activation of CREB and p90RSK in human epileptic hippocampus. Exp Mol Med 2003; 35: 365-370.

17. Bach ME, Barad M, Son H, Zhuo M, Lu YF, Shih R et al. Age-related defects in spatia memory are correlated with defects in the late phase of hippocampal long-term potentiation in vitro and are attenuated by drugs that enhance the CAMP signaling pathway. Proc Natl Acad Sci USA 1999; 96: 5280-5285.

18. Lonze BE, Ginty DD. Function and regulation of CREB family transcription factors in the nervous system. Neuron 2002; 35: 605-623.

19. Turmaine M, Raza A, Mahal A, Mangiarini L, Bates GP, Davies SW. Nonapoptotic neurodegeneration in a transgenic mouse model of Huntington's disease. Proc Natl Acad Sci USA 2000; 97: 8093-8097.

20. Leist $M$, Jaattela $M$. Four deaths and a funeral: from caspases to alternative mechanisms. Nat Rev Mol Cell Biol 2001; 2: 589-598.

21. Ishimaru MJ, Ikonomidou C, Tenkova TI, Der TC, Dikranian K, Sesma MA et al. Distinguishing excitotoxic from apoptotic neurodegeneration in the developing rat brain. J Comp Neurol 1999; 408: 461-476.

22. Shimohata M, Shimohata T, Igarashi S, Naruse S, Tsuji S. Interference of CREBdependent transcriptional activation by expanded polyglutamine stretches - augmentation of transcriptional activation as a potential therapeutic strategy for polyglutamine diseases. J Neurochem 2005; 93: 654-663.

23. Gong B, Vitolo OV, Trinchese F, Liu S, Shelanski M, Arancio O. Persistent improvement in synaptic and cognitive functions in an Alzheimer mouse model after rolipram treatment. J Clin Invest 2004; 114: 1624-1634.
24. Brooks-Kayal AR, Raol YH, Russek SJ. Alteration of epileptogenesis genes. Neurotherapeutics 2009; 6: 312-318.

25. Brightwell JJ, Gallagher M, Colombo PJ. Hippocampal CREB1 but not CREB2 is decreased in aged rats with spatial memory impairments. Neurobiol Learn Mem 2004; 81: $19-26$

26. Tomobe $\mathrm{K}$, Okuma $Y$, Nomura $Y$. Impairment of CREB phosphorylation in the hippocampa CA1 region of the senescence-accelerated mouse (SAM) P8. Brain Res 2007; 1141: 214-217.

27. Porte $\mathrm{Y}$, Buhot MC, Mons N. Alteration of CREB phosphorylation and spatial memory deficits in aged 129T2/Sv mice. Neurobiol Aging 2008; 29: 1533-1546.

28. Qiao X, Lu JY, Hofmann SL. Gene expression profiling in a mouse model of infantile neuronal ceroid lipofuscinosis reveals upregulation of immediate early genes and mediators of the inflammatory response. BMC Neurosci 2007; 8: 95

29. Haltia M. The neuronal ceroid-lipofuscinoses: from past to present. Biochim Biophys Acta 2006; 1762: 850-856.

30. Masedunskas A, King JA, Tan F, Cochran R, Stevens T, Sviridov D et al. Activated leukocyte cell adhesion molecule is a component of the endothelial junction involved in transendothelial monocyte migration. FEBS Lett 2006; 580: 2637-2645.

31. Rudd CE, Taylor A, Schneider H. CD28 and CTLA-4 coreceptor expression and signal transduction. Immunol Rev 2009; 229: 12-26.

32. Bonifati DM, Kishore U. Role of complement in neurodegeneration and neuroinflammation. Mol Immunol 2007; 44: 999-1010.

33. Barco A, Patterson S, Alarcon JM, Gromova P, Mata-Roig M, Morozov A et al. Gene expression profiling of facilitated L-LTP in VP16-CREB mice reveals that BDNF is critical for the maintenance of LTP and its synaptic capture. Neuron 2005; 48: 123-137.

34. Huh GS, Boulanger LM, Du H, Riquelme PA, Brotz TM, Shatz CJ. Functional requirement for class I MHC in CNS development and plasticity. Science 2000; 290: 2155-2159.

35. Alexander JJ, Anderson AJ, Barnum SR, Stevens B, Tenner AJ. The complement cascade: Yin-Yang in neuroinflammation - neuro-protection and -degeneration. J Neurochem 2008; 107: 1169-1187.

36. Gao Y, Deng K, Hou J, Bryson JB, Barco A, Nikulina E et al. Activated CREB is sufficient to overcome inhibitors in myelin and promote spinal axon regeneration in vivo. Neuron 2004; 44: 609-621.

37. Deng W, Neve RL, Rosenberg PA, Volpe JJ, Jensen FE. AMPA receptor subunit composition and CREB regulate oligodendrocyte excitotoxicity. J Biol Chem 2006; 281 36004-36011.

38. Kitagawa K. CREB and CAMP response element-mediated gene expression in the ischemic brain. FEBS J 2007; 274: 3210-3217.

39. Choi YS, Lee B, Cho HY, Reyes IB, Pu XA, Saido TC et al. CREB is a key regulator of striatal vulnerability in chemical and genetic models of Huntington's Disease. Neurobiol Dis 2009; 36: 256-268

40. Viosca J, Malleret G, Bourtchouladze R, Benito E, Vronskava S, Kandel ER et al. Chronic enhancement of CREB activity in the hippocampus interferes with the retrieval of spatial information. Learn Mem 2009; 16: 198-209.

\section{Supplementary Information accompanies the paper on Cell Death and Differentiation website (http://www.nature.com/cdd)}

